\title{
RESEARCH
}

Open Access

\section{Effect of NAC treatment and physical activity on neuroinflammation in subchronic Parkinsonism; is physical activity essential?}

\author{
Ana-Luisa Gil-Martínez ${ }^{1,2}$, Lorena Cuenca ${ }^{1,2}$, Consuelo Sánchez ${ }^{1,2}$, Cristina Estrada ${ }^{1,2}$, \\ Emiliano Fernández-Villalba ${ }^{1,2}$ and María Trinidad Herrero ${ }^{1,2^{*}}$
}

\begin{abstract}
Background: Neuroprotective strategies are becoming relevant to slow down dopaminergic cell death and inflammatory processes related to the progressive neurodegeneration in Parkinson's disease (PD). Interestingly, among others, physical activity (PA) or anti-oxidant agents (such as N-acetyl-L-cysteine, NAC) are common therapeutic strategies. Therefore, this study aims to analyze if there is a synergistic effect of physical activity along with NAC treatment on dopaminergic degeneration and neuroinflammatory response in a 1-methyl-4phenyl-1,2,3,6-tetrahydropyridine (MPTP)-induced Parkinsonism model after subchronic intoxication.

Methods: To ascertain this possibility, 48 8-week-old male mice (C57BL/6 strain) were used. Twenty four of them were placed individually in cages where voluntary physical activity was automatically monitored during 30 days and were divided into groups: (i) control; (ii) NAC; (iii) MPTP, and (iv) MPTP+NAC. The other 24 mice were divided into the same four groups but without physical activity.

Results: The data collected during the treatment period showed that there was an overall increase in the total running distance in all groups under physical activity, including Parkinsonian animals. However, the monitoring data per day showed that the activity routine by MPTP and MPTP+NAC groups was disrupted by alterations in the circardian rhythm because of MPTP intoxication. Results from post-mortem studies in the substantia nigra pars compacta (SNpc) showed significant decrease in the number of TH+ cells in all MPTP groups. Moreover, TH + expression in the striatum was significantly decreased in all MPTP groups. Thus, PA + NAC treatment do not protect dopaminergic neurons against a subchronic intoxication of MPTP. Regarding glial response, the results obtained from microglial analysis do not show significant increase in the number of Iba-1+ cell in MPTP+NAC and MPTP+PA + NAC. In the striatum, a significant decrease is observed only in the MPTP+NAC group compared with that of the MPTP group. The microglial results are reinforced by those obtained from the analysis of astroglial response, in which a decrease in the expression of GFAP+ cells are observed in MPTP+NAC and MPTP $+P A+N A C$ compared with MPTP groups both in the SNpc and in the striatum. Finally, from the study of the astroglial response by the co-localization of GFAP/S100b, we described some expression patterns observed based on the severity of the damage produced by the MPTP intoxication in the different treated groups.

(Continued on next page)
\end{abstract}

\footnotetext{
* Correspondence: mtherrer@um.es

'Department of Human Anatomy and Psychobiology, Clinical and

Experimental Neuroscience Group (NiCE-IMIB), Institute for Aging Research,

School of Medicine, University of Murcia, Murcia, Spain

${ }^{2}$ Biomedical Research Institute of Murcia (IMIB-Arrixaca), Campus of Health

Sciences, University of Murcia, Murcia, Spain
}

(c) The Author(s). 2018 Open Access This article is distributed under the terms of the Creative Commons Attribution 4.0 International License (http://creativecommons.org/licenses/by/4.0/), which permits unrestricted use, distribution, and reproduction in any medium, provided you give appropriate credit to the original author(s) and the source, provide a link to the Creative Commons license, and indicate if changes were made. The Creative Commons Public Domain Dedication waiver (http://creativecommons.org/publicdomain/zero/1.0/) applies to the data made available in this article, unless otherwise stated. 


\begin{abstract}
(Continued from previous page)
Conclusions: These results suggest that the combination of physical activity with an anti-oxidant agent does not have a synergistic neuroprotective effect in the nigrostriatal pathway. Our results show a potential positive effect, only due to NAC treatment, on the neuroinflammatory response after subchronic MPTP intoxication. Thus, physical activity is not essential, under these conditions. However, we believe that physical activity, used for therapeutic purposes, has a beneficial long-term effect. In this line, these results open the door to design longer studies to demonstrate its promising effect as neuroprotective strategy.
\end{abstract}

Keywords: Parkinsonism, Neuroinflammation, Oxidative stress, Physical activity, Microglia, Astrocytes, S100b

\section{Main points}

Synergistic effect of combined treatment based on physical activity and NAC administration (i) is not enough to decrease dopaminergic neuronal death after MPTP intoxication in SNpc and striatum, and (ii) NAC treatment significantly decrease glial response in subchronic MPTP administration.

\section{Background}

Chronic neuroinflammation and oxidative stress processes are features underlying the development and progression of neurodegenerative disorders. In particular, Parkinson's disease (PD) is defined by a clinical picture that consists in impaired motor functions and cognitive deficits caused by the progressive loss of dopaminergic neuronal cells in the substantia nigra pars compacta (SNpc) that leads to the depletion of dopamine fibers in the striatum $[1,2]$ and other catecholaminergic systems [3]. Since the current pharmacological treatments for PD are being controlled in order to reduce motor symptoms [4], new studies are focused on the design of non-pharmacological strategies to avoid side effects caused by drug administration [5].

In this line, physical activity (PA) and exercise attenuate the activation of inflammatory processes in neurological diseases [6]. Although physical activity is defined as "any corporal movement produced by skeletal muscles that requires energy burning" and, exercise as "planned physical activity focused on improving physical abilities" [7], both have similar overall beneficial impact on the body (cardiovascular system, metabolic function, or muscle tone). Additionally, PA can take part in brain functions by improving mood, mental health, and cognitive activity by immune system-mediated effects [8]. Additionally, regular and moderate physical activity maintains the activation of glial cells within a healthy range, which may reduce or delay the progression of brain neurodegeneration [9-14].

Oxidative stress is also a common hallmark in the pathogenesis of PD [15]. It has been described that environmental toxins, such as 1-methyl-4-phenyl-1,2,3,6-tetrahydropyridine (MPTP), contribute to Parkinsonism by increasing oxidative stress in dopaminergic neurons by mitochondrial dysfunction [16]. Several therapeutic strategies, based on antioxidants agents, have been designed to prevent the impact of oxidative processes on the development and progression of neurodegenerative disorders [17]. $\mathrm{N}$-acetyl-L-cysteine (NAC) is a glutathione precursor currently tested for its neuroprotective properties in PD patients ( [18] Identifier: NCT01470027). NAC is a well-stablished antioxidant drug that reduces the loss of dopaminergic neurons and protects against MPTP intoxication in mice [19].

However, since the physical activity and NAC do not protect the $100 \%$ of the dopaminergic cells after MPTP intoxication, we attempted to devise a combined therapy based on NAC, as an anti-oxidant agent, and physical activity, as a non-pharmacological treatment. Through this strategy, we proposed to analyze the possible synergistic effect on dopaminergic neuronal death and on glial activation after MPTP subchronic intoxication in mice.

\section{Methods \\ Animals}

The study was performed on 48 adult male C57BL/6N mice (3 months of age, weight 25-28 g) purchased from Charles River Laboratories (Charles River Laboratories Inc., Barcelona, Spain). Animals were housed in a room with regulated temperature $\left(20 \pm 2{ }^{\circ} \mathrm{C}\right)$ on a 12-h light/ dark cycle, with ad libitum access to food and water. All experiments were performed according to the European Directive 2010/63/UE and the Spanish RD/53/2013 for the protection of animals used for experimentation and other scientific purposes. All the experimental methods and procedures were approved by Institutional Committee on Animal Ethics of the University of Murcia (REGA ES300305440012).

\section{Experimental design and treatments}

All animals were randomly distributed into two main groups (No-MPTP and MPTP), and these turned into 4 other subgroups $(n=6$, each) that received their respective treatment: (i) control; (ii) physical activity (PA); (iii) $\mathrm{N}$-acetyl-L-cysteine (NAC), and (iv) PA + NAC (Fig. 1). All in vivo experiments lasted 30 days in which the treatments were performed, and PA was automatically recorded since day 1 after the first 


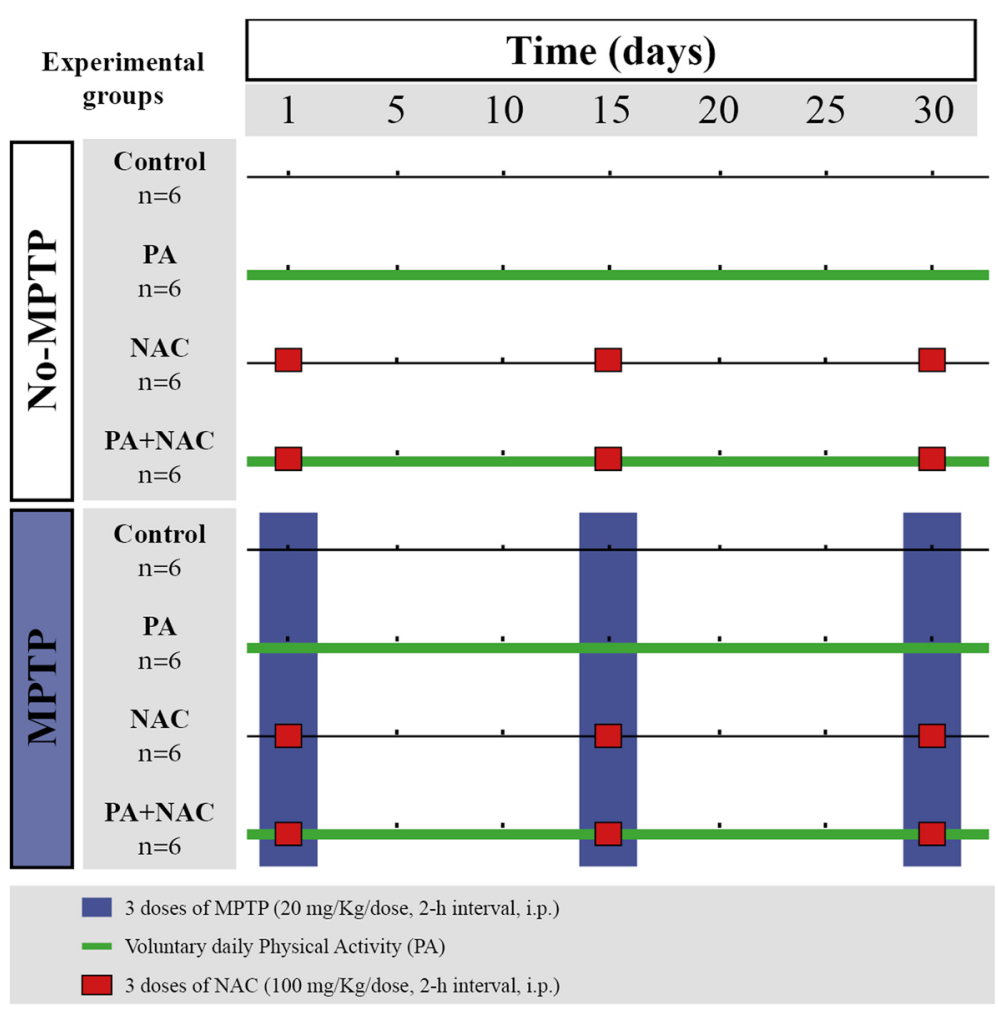

Fig. 1 Scheme of the experimental design with the animal distribution and treatments information

MPTP administration until day 30. PA animals were subjected to daily voluntary physical activity by rotating wheels installed in their individual cages (from days 0 to 30). NAC animals received $N$-acetyl-L-cysteine (NAC, Sigma) (100 mg/kg/dose, 3 doses, 2-h interval) $30 \mathrm{~min}$ prior to each MPTP injection at days 0,15 , and 30 [19].

\section{MPTP administration regime}

Parkinsonism was induced to MPTP groups via intraperitoneal (i.p.) injections of 1-Methyl-4-phenyl-1,2,3,6-tetrahydropyridine hydrochloride (MPTP, Sigma-Aldrich) (20 $\mathrm{mg} / \mathrm{Kg} /$ dose, 3 doses, 2-h interval), dissolved in saline, over a day at days 0,15 and 30 [20, 21]. After MPTP intoxication, mice remained isolated in a room for $48 \mathrm{~h}$ following the safety protocol established for MPTP treatment [22]. No-MPTP animals were treated with vehicle (saline) with the same protocol followed for MPTP injections.

\section{Physical activity monitoring}

Analysis of PA was carried out by a monitored system which measured the complete laps given by each mouse on the wheel per minute during $24 \mathrm{~h}$. A reflective sticker was placed on each wheel, so that the count of laps was based in the reflecting point detected by a photosensitive sensor. This evaluation system aimed to observe the development and progression of the symptoms of both intoxicated and control mice (Fig. 2a). The results from the physical activity monitorization are expressed as running distance in $\mathrm{km}$.

\section{Tissue collection and specific staining}

$48 \mathrm{~h}$ after the last MPTP injection, mice were intraperitoneally anesthetized with a mixture of Xylazine $(50 \mathrm{mg} /$ $\mathrm{kg}$, Xilagesic 2\%, Calier Laboratories) and Ketamine (Imagene $50 \mathrm{mg} / \mathrm{kg}$, Merial) mixture at a ratio of 1:1. The brains were immediately removed and fixed with $4 \%$ paraformaldehyde in phosphate-buffered saline (PBS, $0.1 \mathrm{M}, \mathrm{pH} 7.4$ ) and stored at $4{ }^{\circ} \mathrm{C}$. After $48 \mathrm{~h}$, samples were washed with $\mathrm{PBS}$ and absolute ethanol and were embedded in paraffin blocks. The striatum and mesencephalon were cut in coronal sections (thickness $7 \mu \mathrm{m}$ ) on a microtome (Thermo Scientific HM 325 Rotary Microtome, Thermo Fisher Scientific).

Series of sections were stained with tyrosine hydroxylase $(\mathrm{TH})$ (mouse polyclonal antibody), ionized calcium-binding adapter molecule 1 (Iba-1) (rabbit polyclonal antibody), glial fibrillary acid protein (GFAP) (mouse polyclonal antibody), and (low molecular) calcium-binding protein $\mathrm{b}(\mathrm{S} 100 \mathrm{~b})$ (rabbit monoclonal antibody). 

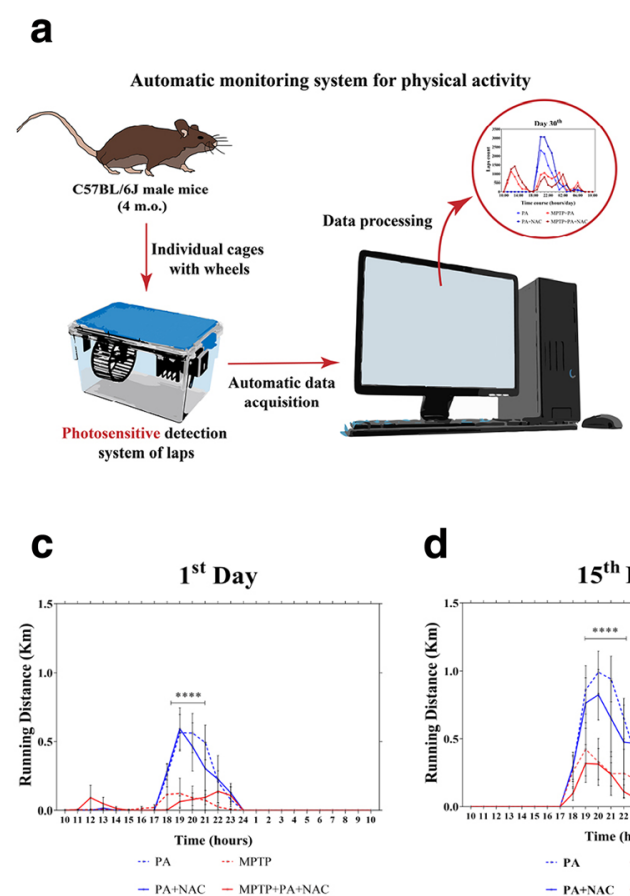

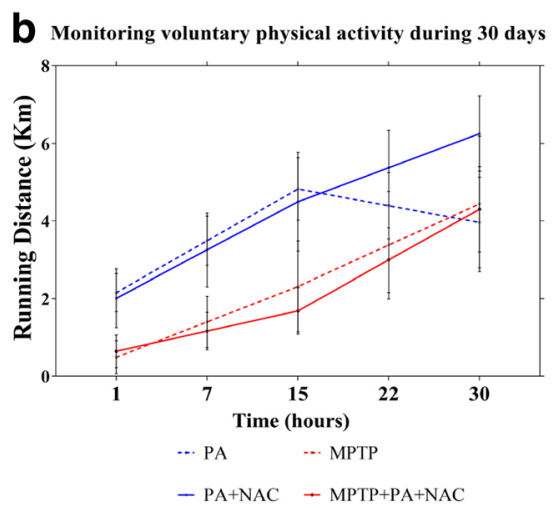

d $15^{\text {th }}$ Day

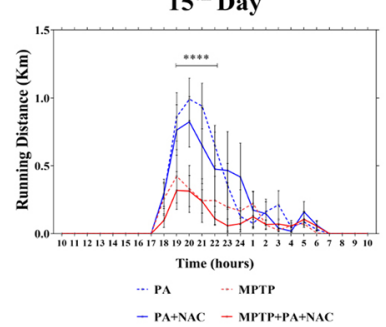

e

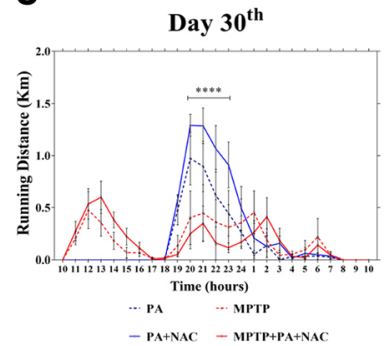

Fig. 2 Monitorization of the daily voluntary physical activity. a) Scheme of the methodology for the automatic monitoring system for physical activity. b) Total running distance ( $\mathrm{Km}$ ) of the different experimental groups during the experiment. It can be observed that No-MPTP mice reach a stable activity on day 15 of the treatment and MPTP treated mice peaked more slowly. c-e Total running distance (km) through $24 \mathrm{~h}$ of the different groups on day 1 (c), day 15 (d), and 30 (e)

\section{Immunohistochemistry and immunofluorescence}

For $D A B$ detection. The sections were deparaffinized in $\mathrm{xy}-$ lene, rehydrated in a decreasing ethanol gradient $(100 \%$, $95 \%$, and $80 \%$ ) and distilled water. Antigen retrieval was performed with citrate buffer (10 mM citric acid, $\mathrm{pH}$ 6.0) for $30 \mathrm{~min}$ at $95{ }^{\circ} \mathrm{C}$ with constant shaking. Endogenous peroxidase activity was inhibited with $0.3 \% \mathrm{H}_{2} \mathrm{O}_{2}$ for $20 \mathrm{~min}$ and non-specific Fc binding sites were blocked with $10 \%$ goat serum for $1 \mathrm{~h}$. The sections were incubated overnight $\left(4^{\circ} \mathrm{C}\right.$, constant shaking) with primary antibody diluted (Table 1$)$ in TBS $1 \times(0.1 \mathrm{M}, \mathrm{pH} 8.4)$ containing $1 \%$ goat serum and $0.5 \%$ Triton X-100. The sections were incubated for $30 \mathrm{~min}$ in secondary antibody diluted (Table 1) in TBS $1 \times$. Binding of antibody was detected with avidin-biotin conjugated to peroxidase (ABC Elite Kit, Vectastain, Vector Labs). The sections were dehydrated in graded ethanol series $(80 \%, 95 \%$, and 100\%) and in xylene before being cover-slipped [20].

For immunofluorescence detection. The sections were treated with citrate buffer $(10 \mathrm{mM}$, citric acid, $\mathrm{pH}$ 6.0) for $1 \mathrm{~h}$ at $95{ }^{\circ} \mathrm{C}$ and non-specific $\mathrm{Fc}$ binding were blocked with $2.5 \%$ goat serum for $1 \mathrm{~h}$. The sections were incubated overnight $\left(4{ }^{\circ} \mathrm{C}\right.$, constant shaking) with primary antibody diluted (Table 1 ) in TBS $1 \times$ containing $2.5 \%$ goat serum and $0.5 \%$ Triton $\mathrm{X}-100$. The

Table 1 Dilutions for primary and secondary antibodies used for immunohistochemistry and immunofluorescence

\begin{tabular}{|c|c|c|c|c|c|}
\hline Primary antibodies, code & Host & $\mathrm{IHQ} / \mathrm{IF}$ & Dilution, incubation time & Secondary antibodies, code & Dilution, incubation time \\
\hline \multirow[t]{2}{*}{ Anti-TH } & Mouse, MAB318, Millipore & $\mathrm{IHQ}$ & 1:500, o.v. & $\begin{array}{l}\text { Anti-lgG mouse (Biotinylated), } \\
\text { BA-9200 }\end{array}$ & $1: 250,1 \mathrm{~h}$ \\
\hline & & IF & 1:500, o.v. & Double Labeling Kit, DK8818ª & $30 \mathrm{~min}$ \\
\hline \multirow[t]{2}{*}{ Anti-Iba1 } & Rabbit, B178846, Abcam & $\mathrm{IHQ}$ & 1:1000, o.v. & $\begin{array}{l}\text { Anti-lgG rabbit (Biotinylated) } \\
\text { BA-1000 }\end{array}$ & $1: 250,1 \mathrm{~h}$ \\
\hline & & IF & 1:1000, o.v. & Double Labeling Kit, DK8818 & $30 \mathrm{~min}$ \\
\hline \multirow[t]{2}{*}{ Anti-GFAP } & $\begin{array}{l}\text { Mouse, MAB360, } \\
\text { Millipore }\end{array}$ & $\mathrm{IHQ}$ & 1:500, o.v. & $\begin{array}{l}\text { Anti-lgG mouse (Biotinylated) } \\
\text { BA-9200 }\end{array}$ & $1: 250,1 \mathrm{~h}$ \\
\hline & & IF & 1:500, o.v. & Double Labeling Kit, DK8818 & $30 \mathrm{~min}$ \\
\hline Anti-S100b & Rabbit, AB52642, Abcam & IF & 1:500, o.v. & Double Labeling Kit, DK8818 & $30 \mathrm{~min}$ \\
\hline
\end{tabular}


sections were incubated for $30 \mathrm{~min}$ in darkness in secondary antibody ready-to-use kit (Double Labeling Kit, Vector Laboratories). The samples were assayed (Vectashield Antifade Mounting Medium, Vector Laboratories).

\section{Quantification and stereological analysis}

The nigrostriatal pathway was defined according to anatomical coordinates of the mouse brain atlas [23] and using the same protocol reported by J. Blesa and colleagues [24] but specifically adjusted to mouse brain [25]. Quantification of DAB-labeled cells were carried out throughout the midbrain [from $-2.06 \mathrm{~mm}$ to 3.80 relative to Bregma], and dopaminergic striatal terminals [from $0.26 \mathrm{~mm}$ to $0.02 \mathrm{~mm}$ relative to Bregma] was performed on series of eight coronal sections from each animal. All figures for immunohistochemical analysis were obtained by Hall 100 ZEISS optical microscope with an Axiocam ZEISS digital camera.

\section{Quantification of TH-positive ( $\mathrm{TH}+$ ) cells}

In $\mathrm{SNpc}$, the total number of $\mathrm{TH}+$ cells was determined using the $\times 20$ objective by counting the number of stained neurons (nucleus were used as counting unit) divided by the delineated area of the SNpc expressed as $\mathrm{TH}+$ cells $/ \mathrm{mm}^{3}$. In the striatum, optical density of $\mathrm{TH}+$ innervations was measured in digitalized images using the NIH Image software (Image); NIH, Bethesda, MD, USA). The optical density of DAB signal in each micrograph was taken with the $\times 40$ objective covering the entire rostro-caudal striatum, expressed as area $(\%) /$ mean gray value.

\section{Quantification of microglial (Iba-1+) cells}

Iba-1-positive cells were counted in SNpc using the same protocol established for TH-labeled samples. In the striatum, the microglia analysis was performed using the $\times 20$ magnification, and Iba- $1+$ cells were counted and expressed as Iba $-1+$ cells $/ \mathrm{mm}^{3}$.

\section{Quantification of astrocytes (GFAP+)}

In SNpc, astrocytic GFAP expression was analyzed with the same protocol established for TH-labeled samples. In the striatum, the astroglial GFAP expression was carried out using the $\times 20$ objective for counting GFAP+ cells and expressed as GFAP+ cells $/ \mathrm{mm}^{3}$.

\section{Confocal analysis and 3D reconstruction}

The brain sections were analyzed using Leica TCS-SP8 confocal microscope with the $\times 40$ oil objective (PL APO $40 \mathrm{X} / 1.3 \mathrm{AN}$ ) and the $\times 63$ glycol objective (PL APO 63X/ $1.3 \mathrm{AN}$ ) and Leica Application Suite X (LAS X, Leica Microsystems). After the immunostaining, confocal images were obtained in sequential acquisition to avoid cross-reactions or photobleaching of fluorophores during long-lasting manual mode. The images were not postprocessed for the analysis. Series of sections were obtained by defining the upper and lower limits using the $\mathrm{Z}$ position covering $20 \mathrm{~mm}$ of thickness of the brain sample with $0.5 \mathrm{~mm}$ thickness per optical section. We show higher magnification images to observe with better detail that the microglial and astroglial morphological features and other aspects, which were not due to invasion or cross-reaction of the fluorescence from other channels. FIJI-ImageJ software was used for the composition of three-dimensional reconstructions from the stacks of confocal images.

\section{Data and statistical analysis}

Data were presented as mean \pm SD and were analyzed by means of a two-way analysis of variance test (two-way ANOVA) with a Sidak post hoc analysis for multiple group comparisons. The null hypothesis was rejected at a significance level of 0.05 so that differences with a value of $p<0.05$ were considered significant and with a value of $p<0.01$ were considered as statistically very significant. All statistical analyses were conducted using GraphPrism7 software (GraphPad Software Inc.). A detailed description of the statistical analysis can be found in the Additional file 1 .

\section{Figures and artwork}

Schematics, tables, and vector graphics were created using Adobe Illustrator CS6, and figures are arranged and compiled using Adobe InDesign CS6.

\section{Results}

Voluntary PA increased through the experiment in both No-MPTP and MPTP groups

Voluntary physical activity was automatically monitorized during 30 days recording the number of laps performed by each mice expressed as running distance. In Fig. $2 b$, it is shown that the total running distance increased in all groups since the start of the experiment. A two-way ANOVA (time $\times$ groups) was performed and the results indicated a significant main effect for Time $[F(4,80)=$ 25.61, $p<0.0001]$ but not for Groups $[F[3,20]=25.61, p$ $<0.0001]$. Following this data, we performed a simple effects within columns. Both PA and PA + NAC groups significantly increase $(p<0.05)$ the running distance from day 15 compared with day 1 . In addition, physical activity was also significantly increase for Parkinsonian mice since the beginning but with a shorter mean running distance compared with No-MPTP animals.

In Fig. $2 \mathrm{c}-\mathrm{e}$, the monitorization of the physical activity over $24 \mathrm{~h}$ in different days is shown. The data was analysis by repeated measures two-way ANOVA (time $\times$ groups) that revealed a significant interaction on day $1[F(72,480)=3.337, p<0.0001]$, day $15[F(72$, 
$480)=2.002, p<0.0001]$, and day $30[F(72,480)=$ $4.412, p<0.0001]$. Following this data, we proceeded to analyze simple effects within columns with a Sidak's as multiple comparisons post hoc test. On day 1, the most significantly peak of activity was registered from 18:00 to 21:00 performed by PA $(p<0.0001)$ and PA + NAC $(p<0.0001)$. For MPTP intoxicated mice there was no significant peak of physical activity registered. On day 15, it is repeated the same pattern of significant peak of physical activity from 18:00 to 22:00 performed by PA $(p<0.0001)$ and PA + NAC $(p<0.0001)$ but not significant peak of running distance was performed by MPTP intoxicated mice. Finally, on day 30, the significant peak of physical activity was performed from $19: 00$ to $23: 00$ by PA $(p<0.0001)$ and PA + NAC $(p<0.0001)$ and not significant peak of running distance for Parkinsonian animals. These data show that the total running distance over 1 day does not follow a normal circardian rhythm in MPTP groups compared to the No-MPTP groups, which results in a different activity behavior because of MPTP intoxication.

\section{PA + NAC treatment is not enough to protect DA neurons under subchronic MPTP intoxication}

It has been described that in PD, there is a dopaminergic neuronal loss in the $\mathrm{SNpc}$ and in the neuronal striatal innervations. In order to study the possible neuroprotective effect of PA, NAC, and, mainly, the combined PA + NAC treatment on dopaminergic neuronal death caused by subchronic MPTP-injections, we assessed immunohistochemistry for $\mathrm{TH}$ expression in SNpc and striatum (Fig. 3). All Parkinsonized mice suffered a significant loss of $\mathrm{TH}$ expression in dopaminergic neurons compared to their respective No-MPTP groups. A two-way ANOVA (treatments $\times$ Parkinsonism) was performed, and the results indicated a significant main effect for treatments $[F[3,31]=5.1420, p=0.0053]$ and for Parkinsonism $[F[1,31]=156.70, p<0.0001]$. There was no significant interaction between these two variables $[F[3,31]=0.7234, p=0.5457]$ so that they cause an overall main effects (Fig. 3b).

In the same way, dopaminergic striatal innervation was analyzed by optical density measurements of $\mathrm{TH}$-stained slices. The MPTP intoxication induced a significant main effect $[F[1,31]=82.70, p<0.0001]$ in all the groups. However, there is no effect overall by treatments variable $[F[3,31]=0.9184, p=0.4469]$, and the interaction is considered not significant $[F[3,31]=$ 0.6373, $p=0.5983$ ] (Fig. 3c). These data demonstrate that there is a very significant main effect of MPTP intoxication in all groups under subchronic administration that it is not enough by the administration of NAC or/and physical activity.
Effect of PA + NAC treatment on Iba-1 expression in the SNpc and striatum after MPTP intoxication

We studied microglial activation by analysis for Iba-1 labeling, a specific microglial marker which does not cross-react neither with neurons nor astrocytes (Fig. 4). In the SNpc, no significant increase of microglial cells is observed in untreated mice and in MPTP+NAC and $\mathrm{MPTP}+\mathrm{PA}+\mathrm{NAC}$ groups while MPTP $(p<0.0001)$ and MPTP+PA $(p=0.0040)$ groups presented a significant increase in the number of Iba-1+ cells compared to their No-MPTP groups (Fig. 4b). According to these results, MPTP $(p<0.0001)$ and MPTP+PA $(p=0.0348)$ mice showed a significant increase in microglial activation in the striatum compared to their No-MPTP groups; this increment can also be observed in the $\mathrm{MPTP}+\mathrm{PA}+\mathrm{NAC}$ group $(p=0.0082)$, although this microglial cells may have adopted a protective role [26] (Fig. 4c). Reinforcing the results obtained by immunohistochemical analysis, it can be observed in Fig. 4d, morphological characteristics, already described for active microglia [27].

\section{Effect of PA + NAC treatment on GFAP expression in the SNpc and striatum after MPTP intoxication}

Activated astrocytes are characterized by higher GFAP expression levels and increased cell body size (hypertrophy) in the affected brain regions [28]. Quantification of GFAP+ cells in the SNpc showed a significant increase in the MPTP $(p<0.0001)$ and in MPTP+PA $(p=0.0021)$ groups compared with their control groups. In relation to the results described above, no significant differences in astrocytes activation were found in $\mathrm{MPTP}+\mathrm{NAC}$ and $\mathrm{MPTP}+\mathrm{PA}+\mathrm{NAC}$ when compared to the NAC and PA + NAC groups, respectively (Fig. 5b). These findings point out to a decrease in the damage produced by the administration of MPTP when animals are treated with NAC and with the combination of PA $+\mathrm{NAC}$, since a significant decrease in the number of GFAP+ cells is observed in $\mathrm{MPTP}+\mathrm{NAC}(p=0.0008)$ and MPTP+PA + NAC $(p<$ $0.0001)$ compared to the MPTP-treated group. In the striatum, the number of GFAP+ cells was significantly increased in all MPTP groups compared to their respective No-MPTP groups: MPTP $(p<0.0001)$, MPTP $+\mathrm{PA}(p=0.0060), \mathrm{MPTP}+\mathrm{NAC}(p<0.0001)$, and MPTP $+\mathrm{PA}+\mathrm{NAC}(p=0.0002)$. However, although there is a significant increase in the number of astrocytes that express GFAP in all MPTP groups when they are compared with their respective control groups, it is interesting to note that this significant increase in MPTP mice is maintained when compared to Parkinsonian animals treated with PA $(p<0.0001)$, NAC $(p=$ 0.0002), and PA + NAC $(p<0.0001)$ (Fig. 5c). 

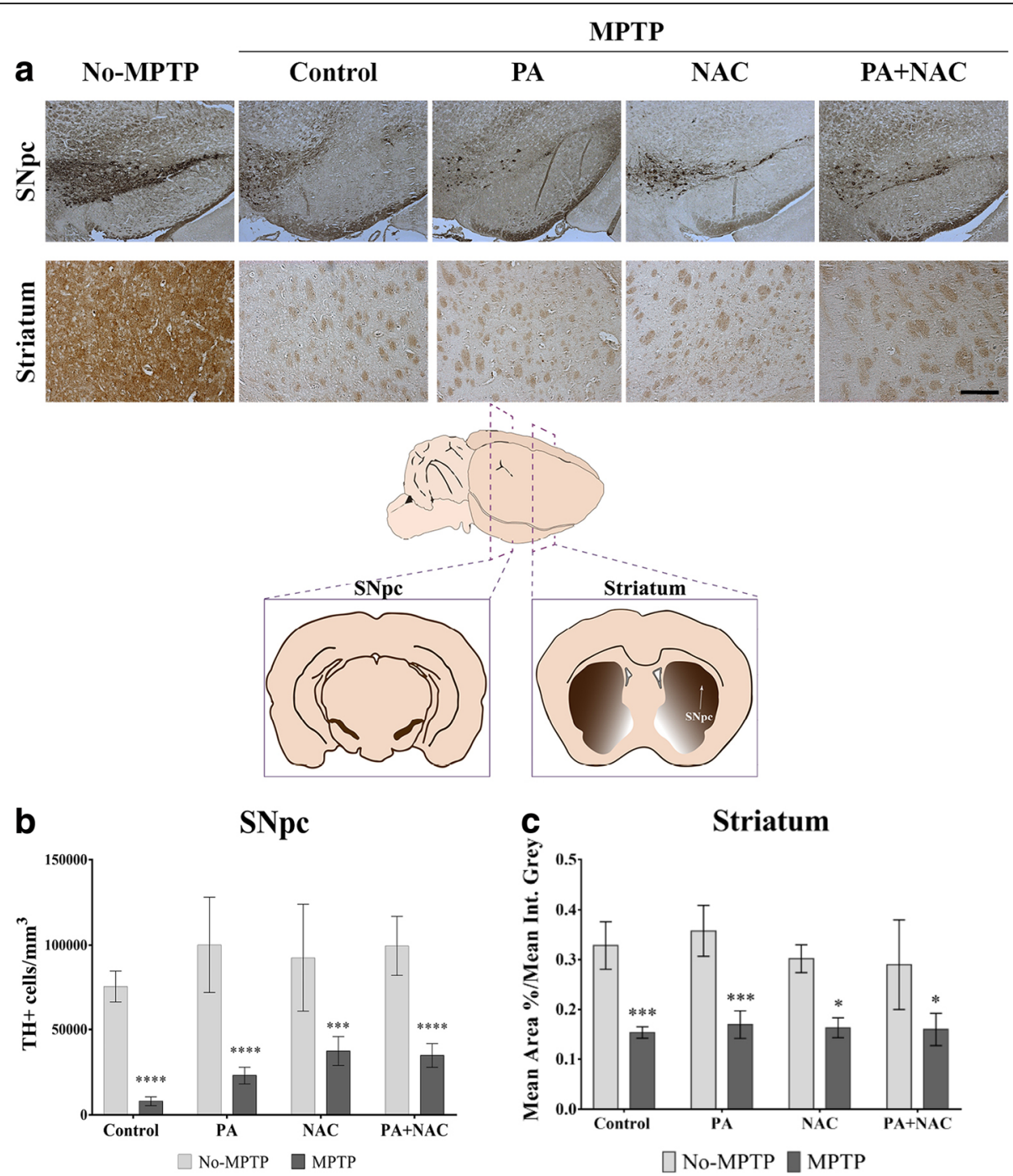

Fig. 3 Quantification of $\mathrm{TH}+$ expression in SNpc and striatum. a Representative images from TH immunolabeling in SNpc and striatum (magnification $\times 10$, scale bar $=100 \mu \mathrm{m})$. b Quantification of TH+ cells $/ \mathrm{mm}^{3}$ in SNpc. Results show that MPTP $(* * * *<0.0001)$, MPTP+PA $\left({ }^{* * *} p=0.0001\right), \operatorname{MPTP}+\mathrm{NAC}\left({ }^{* * *} p=0.0006\right)$, and MPTP+PA + NAC $\left({ }^{* * *} p<0.0001\right)$ have a significant decrease of TH+ cells $/ \mathrm{mm}^{3}$ compared to their No-MPTP groups. c Measurement of TH+ expression in striatal fibers. All MPTP mice have a significant decrease compared with their respective control groups: MPTP $\left({ }^{* *} p=0.0007\right), \mathrm{MPTP}+\mathrm{PA}\left({ }^{* * *} p=0.0004\right), \mathrm{MPTP}+\mathrm{NAC}\left({ }^{*} p=0.0143\right)$, and MPTP $+\mathrm{NAC}+\mathrm{PA}\left({ }^{*} p=0.0396\right)$

\section{GFAP/S100b co-localization in the SNpc and striatum:} different astrocyte profiles based on groups treatment In our study, we described profiles of expression (S100b/GFAP) that were repeated among the individuals of the same treatment group.

Thus, we established six profiles of expression: [1] the expression of S100b is only located in the nucleus and GFAP is not expressed; [2] S100b is expressed in the nucleus and GFAP expression is located in the soma; [3] S100b expression is only located in the nucleus whereas GFAP expression is in the soma and in the processes; [4] S100b is located in the nucleus but, in this case, it is found a double-labeled S100b/GFAP in the soma; [5] same characteristics as state 4, but with double-labeled both in the soma and in the processes; and [6] only S100b positive expression in the soma. In all MPTP-untreated mice, mainly profile [1] was found (Fig. 6). In MPTP and MPTP+PA groups, states 4 and 5 were found; the colocalization between S100b and GFAP is what characterizes these two profiles, which, in turn, could be related to a more severe injury after MPTP administration. Finally, MPTP+NAC and MPTP+PA + NAC groups show 2 and 3 expression profiles, which could be translated to an acute damage.

\section{Discussion}

In this study, the regimen of MPTP administration was defined based on the dynamic changes in the 


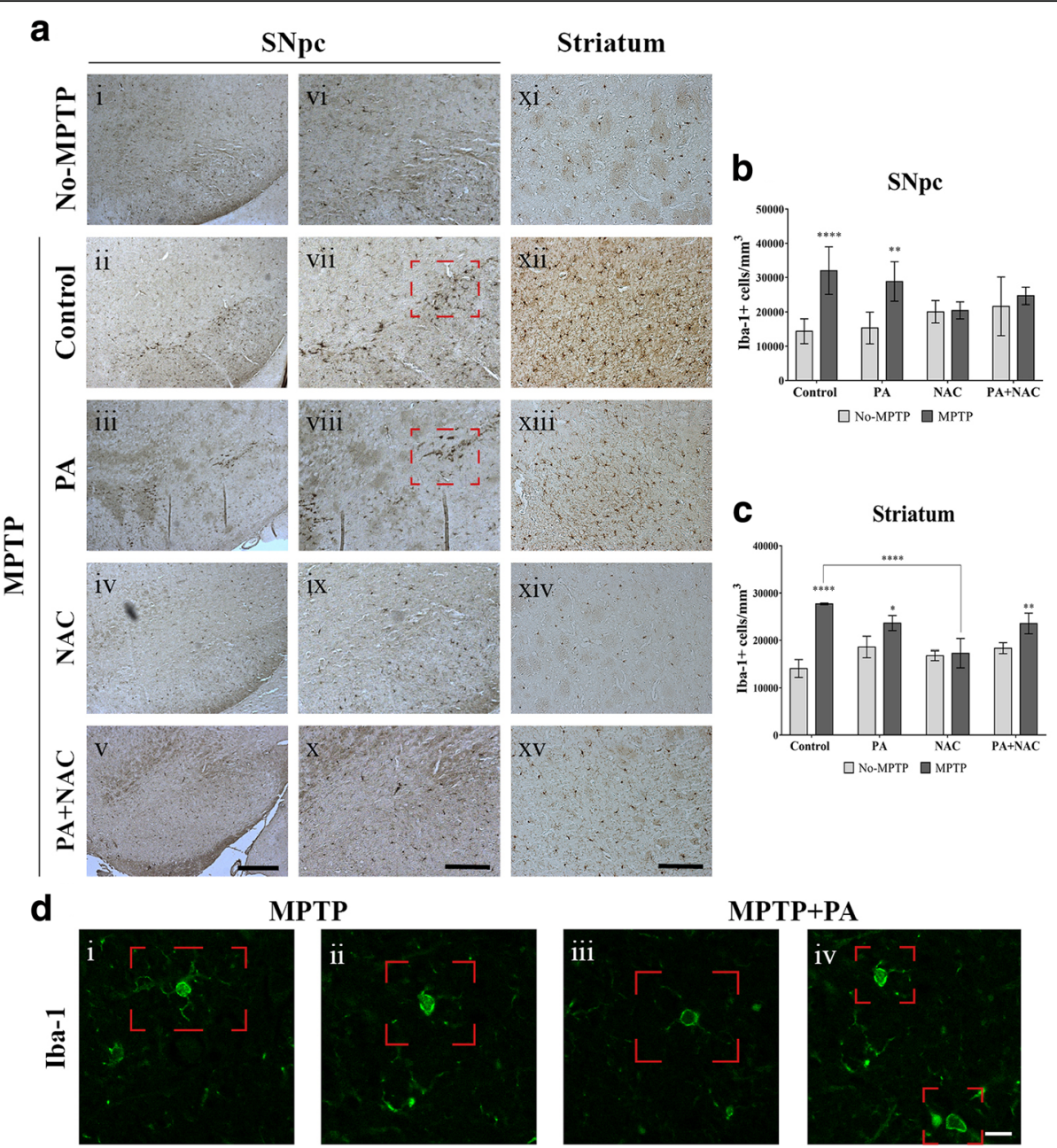

Fig. 4 Microglial cells quantification in the nigrostriatal pathway. a Representative photomicrographs of Iba-1+ expression in SNpc and striatum (magnification $\times 10(\mathrm{i}-\mathrm{v})$ and $\times 20(\mathrm{vi}-\mathrm{xv})$, scale bar $=100 \mu \mathrm{m}$ and $50 \mu \mathrm{m}$, respectively). $\mathbf{b} \mathrm{lba}-1+$ cells density in the SNpc is significantly increase in MPTP $(* * * p<0.0001)$ and MPTP+PA (** $p=0.0040)$ groups compared to their No-MPTP groups. $\mathbf{c}$ In the striatum, microglial cells expression were significantly increase in MPTP (**** $p<0.0001)$, MPTP+PA $\left({ }^{*} p=0.0348\right)$, and MPTP+PA + NAC $\left({ }^{* *} p=0.0082\right)$. Interestingly, it can be observed that significant decrease is found in MPTP+NAC $\left.{ }^{* * * *} p<0.0001\right)$ compared to MPTP group. $\mathbf{d}$ Representative micrographs from immunofluorescence under high magnification showing lba-1+ cells in the SNpc of MPTP (i-ii) and MPTP+PA (iii-iv) groups (magnification $\times 63$, scale bar $=25 \mu \mathrm{m})$

nigrostriatal pathways [29]. We stablished three MPTP administrations (3 doses, at 2-h intervals in 1 day) distributed in three time-points. The first administration was performed on day 1 of the treatment and combined with the administration of NAC before each dose of MPTP [19] in the MPTP+NAC group. The established treatment regime was repeated on the second and third weeks (day 15 and day 30, respectively) after the first MPTP administration. Animals were sacrificed $48 \mathrm{~h}$ after the last injections because it has been described to be the highest peak of glial activation after MPTP injections [21].

Voluntary physical activity was automatically controlled daily in order to analyze the effect on the MPTP intoxication. The results obtained from the total balance of laps per day in the MPTP-treated mice subjected to daily PA showed a stabilization for activity from the beginning to the end of the treatment. It is important to highlight that time has a significant effect on promoting the increase of $\mathrm{PA}$, implying that over the 3-week experiment, both No-MPTP and MPTP groups were more willing to run. Considerably, data obtained from 24-h monitoring presented significant changes between groups, which were accentuated $48 \mathrm{~h}$ after MPTP injections. Thus, the rhythm of activity is different between MPTP and No-MPTP animals, peaks of diurnal and nocturnal activity were observed in the intoxicated groups. These results agree with other studies conducted in Parkinsonized macaques whose motor activities varied throughout the day as a 


\section{a}

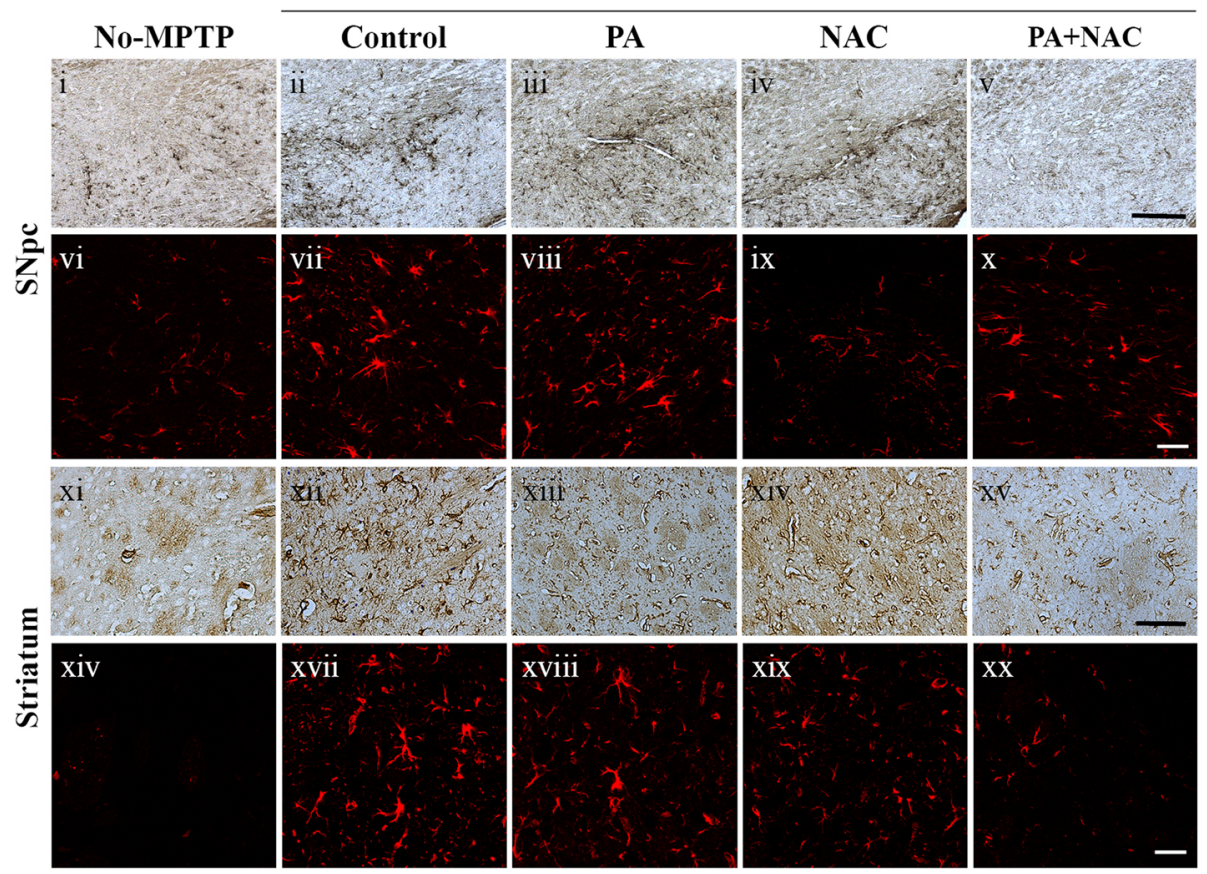

b

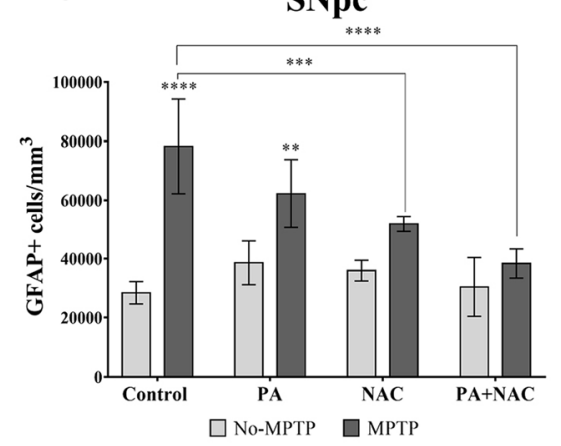

C

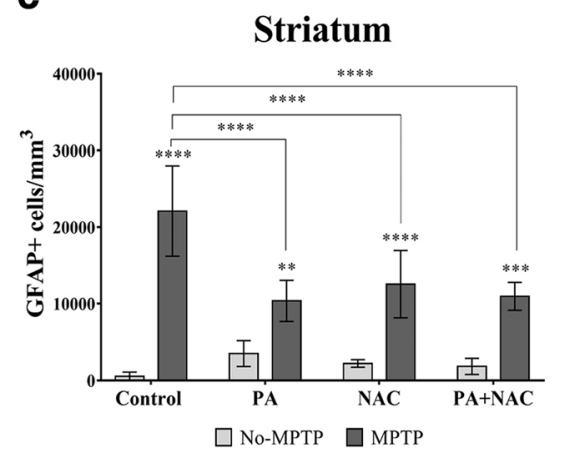

Fig. 5 Effect of combined treatment on GFAP+ expression in the SNpc and striatum. a Images from immunolabeling of GFAP in the SNpc and in the striatum (magnification $\times 20$ ( $i-v$ and $x i-x v$ ), scale bar $=50 \mu m$; magnification $\times 63$ (vi-x and $x v i-x x$, scale bar $=25 \mu m$ ). $\mathbf{b}$ GFAP+ cells density in the SNpc is significantly increase in MPTP $\left({ }^{* * *} p<0.0001\right)$ and MPTP+PA $\left({ }^{* * *} p=0.0021\right)$ groups compared to No-MPTP groups. Interestingly, a significant decrease in GFAP+ expression were found comparing MPTP+NAC $\left(* * * p=0.0008\right.$ and MPTP $+P A+N A C\left(*^{* * *} p<0.0001\right)$ with MPTP group. $\mathbf{c}$ In the striatum, MPTP $\left({ }^{* * *} p<0.0001\right)$, MPTP $+P A\left({ }^{* *} p=0.0060\right), M P T P+N A C(* * * *<0.0001)$, and MPTP+PA $+N A C\left({ }^{* * *} p=0.0002\right)$ express significant increase in GFAP+ cells density comparing with their respective No-MPTP groups. As in the SNpc, significant decrease were found between MPTP+PA $\left({ }^{* * *} p<0.0001\right)$, MPTP+NAC $\left({ }^{* * *} p=0.0002\right)$, and MPTP+PA + NAC $\left({ }^{* * * *} p<0.0001\right)$

consequence of an alteration of the circadian rhythm caused by the MPTP intoxication [30, 31].

Both PA and NAC have individually showed to significantly decrease the loss of dopaminergic neurons in MPTP-induced Parkinsonian mice [6, 19]. However, our data showed a significant decrease of $\mathrm{TH}+$ expression both in the SNpc and in the striatum in all MPTP groups. The fact that dopaminergic neurons are not protected against MPTP intoxication by the treatment based on NAC and physical activity can be explained by the experimental design. Regimes known as sub-chronic are, indeed, serial MPTP acute insults over days or weeks. The samples were obtained $48 \mathrm{~h}$ after the last MPTP administration, so we were analyzing the accumulative damage caused by the serial MPTP injections over 3 weeks. Specifically, in the SNpc there is a significant main effect from the treatments, so it may be potentially important to design long-term studies to analyze the recovery capacity of dopaminergic neurons in Parkinsonian mice treated with PA + NAC.

Several investigations suggest that a sustained inflammatory response is a crucial fact for the progression of 

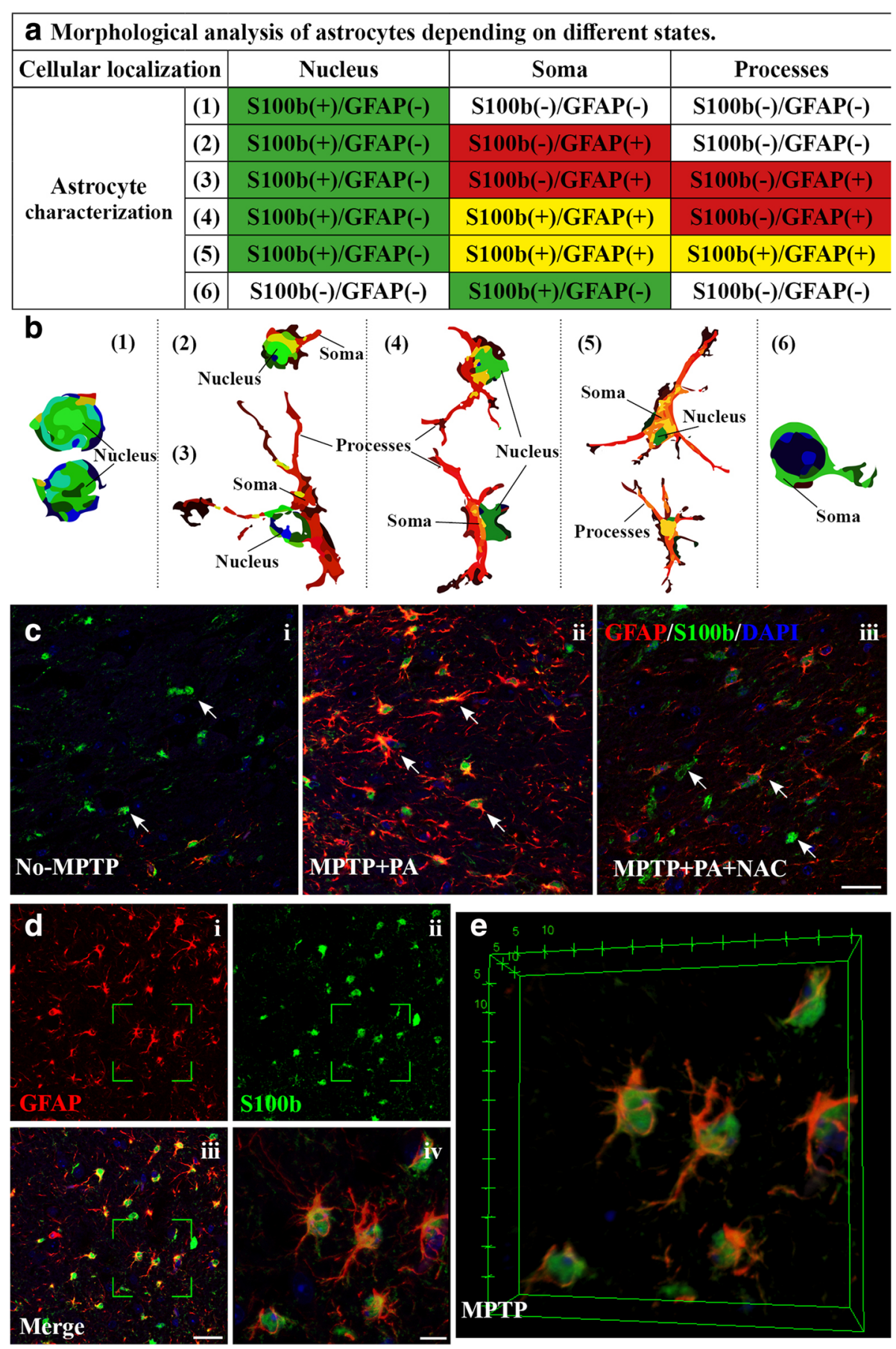

Fig. 6 Description of the observed cellular location profiles of GFAP and S100b in astrocytes. a Descriptive table with the details of the different cellular location profiles. b Cartoons from 1 to 6 illustrate the different location profiles described in the table. $\mathbf{c}$ Representative images of the experimental groups: No-MPTP, MPTP+PA, and MPTP+PA + NAC where these expression profiles are observed. $\mathbf{d}$ Frame showing the separate channels, GFAP (i) and S100b (ii), merge (iii) and 3D projection from z-stack images (iv) in which showed a selective area at high magnification. e 3D reconstruction images to show how it is observed GFAP, S100b, and colocalization of both proteins. (magnification $\times 63$, zoom set at 1 , scale bar $=25 \mu \mathrm{m}$ )

neuronal depletion in PD [32]. Inflammation in the central nervous system (CNS) involves the intervention of a specialized response by activated glial cells and oxidative stress mechanisms [33, 34]. These mainly include microglia, the major resident immune cells in the brain, and astrocytes, the supportive glial cells 
components in neuronal tissue. After an acute injury, glial cells become activated (reactive gliosis) and work to repair the damage and restore cerebral homeostasis. Recent works show that PA has emerged as a potent nonpharmacological component with immune-modifying properties, resulting in an overall whole-body anti-inflammatory effect [6]. Thus, it has been reported that endurance exercise has a neuroprotective effect against dopamine loss and proinflammatory cytokines production by modulating TLR2/MyD88/NF-kB activation, and it also may be implicated in downregulation of a-synuclein [35].

In this work, we analyzed the dynamics interplay between microglia and astroglial cells under different therapeutic conditions in Parkinsonian mice. In the first instance, microglial cells proceed with an anti-inflammatory phenotype with neuroprotective effects [29]. If the initial acute damage is not resolved, a maintained inflammatory response is established and affects surrounding glial cells and neurons promoting a detrimental progression of the disease [36]. In fact, microglial cells' quantification showed a significant increase in the expression of Iba-1 in all MPTP intoxicated groups, with the exception of Parkinsonian mice treated with NAC and PA + NAC in the SNpc. In the striatum, Iba-1+ expression was significantly increased only in MPTP and MPTP+PA groups compared with their No-MPTP groups, and no significant changes were observed in $\mathrm{MPTP}+\mathrm{NAC}$ and MPTP+PA + NAC. In the striatum, Iba-1+ cells were significantly increased in MPTP and MPTP+PA compared with their No-MPTP groups.

Reactive astrogliosis is triggered by active microglia [37] or dopaminergic neuronal death [38]. In the SNpc, our data showed that GFAP+ expression was significantly increased in MPTP and MPTP+PA groups while no significant results were found in MPTP $+\mathrm{NAC}$ and $\mathrm{MPTP}+\mathrm{PA}+$ NAC compared to their No-MPTP groups. These data reinforce the results obtained for microglia activation.

Although the literature does not reach a consensus in the description and identification of the morphological characteristics of activated glial cells, some features are undoubted. Activated microglial cells acquire a heterogeneous forms from ramified to amoeboid macrophage-like appearance [39]. In this line, changes in reactive astrocytes depend on the severity of the aggression including progressive alterations in molecular expression, cell hypertrophy, and in severe cases, proliferation and scar formation [40]. In this study, we observed these profiles of morphological changes both in the SNpc and in the striatum in those groups that were more damaged by the MPTP intoxication (Figures 5 (vi-x and $\mathrm{xi}-\mathrm{xv})$ ).

Finally, we studied S100b, a protein mainly expressed in astrocytes with an important role in the initial phase of brain insults as well as maintenance of glial-mediated pro-inflammatory state. Its release by astroglia varies depending on different factors as TNF- $\alpha$ in degenerative disorders [41]. In the present study, different GFAP and S100b expression profiles were defined based on the observations of the dual immunolabeling. We propose two possible situations regarding immunofluorescence analysis; one of them is the possibility of observing subpopulations of astrocytes that are evidenced by different colocalization profiles of GFAP and S100b [42]. However, our interpretation of the results fits better with a second proposed situation that supports that different colocalization profiles change depending on the severity of the injury. Thus, as it has already been shown, S100b would be released by GFAP+ and GFAP- astrocytes may play an important role in the development of MPTP-induced dopaminergic neurodegeneration in mice [43]. In this study, we mainly found that S100b is located in the nucleus of GFAP- astrocytes in No-MPTP, MPTP+NAC, and MPTP+PA + NAC groups, which showed to be the less affected. These results are in accordance with a previous study [43]. Then, we observed an expression of S100b moving from the nucleus to the soma in both GFAP+ and GFAP- astrocytes in groups from least (MPTP $+\mathrm{NAC}$ and $\mathrm{MPTP}+\mathrm{PA}+\mathrm{NAC})$ to most (MPTP and MPTP+PA) damaged animals by MPTP intoxication, respectively. This data could be especially interesting in the identification of the astrocyte's activation state and therefore in identifying the degree of severity under a pathological condition.

\section{Conclusions}

These results suggest that the combination of physical activity with an anti-oxidant agent does not have a synergistic neuroprotective effect in the nigrostriatal pathway after subchronic MPTP intoxication. However, our results show a potential positive effect only due to NAC treatment on the neuroinflamatory response both in the $\mathrm{SNpc}$ and in the striatum after subchronic MPTP intoxication. Thus, concluding that physical activity, under these conditions, is not necessary. However, we believe that physical activity, used for therapeutic purposes, has a beneficial long-term effect. In this line, these results open the door to design longer studies to demonstrate the promising effect of physical activity as neuroprotective strategy.

\section{Additional file}

Additional file 1: Detailed description of the statistical analysis. (DOCX $234 \mathrm{~kb})$

\section{Abbreviations}

CNS: Central nervous system; DA: Dopaminergic; GFAP: Glial fibrillary acidic protein; Iba-1: lonized calcium-binding adapter molecule 1;

IR: Immunoreactive; MPTP: 1-methyl-4-phenyl-1,2,3,6-tetrahydropyridine; NAC: N-acetyl-L-cysteine; PA: Physical activity; PD: Parkinson's disease; SNpc: Substantia nigra pars compacta 


\section{Acknowledgements}

The authors want to thank to Fernando Ruiz Abellán (University of Murcia) for its electronic and technical support in the monitoring system of physical activity.

\section{Funding}

Research work of the Authors was supported by the Spanish Ministry of Science and Innovation (FIS PI13 01293), Fundación Séneca (19540/PI/14) and "Prediction of cognitive properties of new drug candidates for neurodegenerative diseases in early clinical development" (European Community's Seventh Framework Programme (FP7/2007-2013) for the Innovative Medicine Initiative under Grant Agreement No. 115009) to MTH.

\section{Availability of data and materials}

Please contact author for data requests. A representative image of the No-MPTP groups has been shown for each marker, since the results between them are similar. However, the authors have all the images in case of any requirement.

\section{Authors' contributions}

ALGM performed the analysis of the data, prepared the figures, and wrote the manuscript. ALGM and CS carried out all the experiments. LC, CE, and EFV participated in the in vivo and post mortem experiments. MTH conceived the study idea, designed the study, discussed the results, and worked in the manuscript preparation. All authors read and approved the final manuscript.

\section{Ethics approval and consent to participate}

All animal testing methods and procedures were approved by the Council of the European Community Committee (2010/63/EU) and by the Institutional Committee on Animal Ethics of the University of Murcia (REGA ES300305440012).

\section{Consent for publication}

Not applicable.

\section{Competing interests}

The authors declare that they have no competing interests.

\section{Publisher's Note}

Springer Nature remains neutral with regard to jurisdictional claims in published maps and institutional affiliations.

Received: 26 July 2018 Accepted: 31 October 2018

Published online: 26 November 2018

\section{References}

1. Kalia LV, Lang AE. Parkinson's disease. Lancet. 2015;386(9996):896-912.

2. Pérez-Otaño I, Oset C, Luquin MR, Herrero MT, Obeso JA, Del Río J. MPTP-induced parkinsonism in primates: pattern of striatal dopamine loss following acute and chronic administration. Neurosci Lett. 1994; 175(1-2):121-5.

3. Blesa J, Przedborski S. Parkinson's disease: animal models and dopaminergic cell vulnerability. Front Neuroanat. 2014:8:1-12.

4. Li B-D, Bi Z-Y, Liu J-F, Si W-J, Shi Q-Q, Xue L-P, et al. Adverse effects produced by different drugs used in the treatment of Parkinson's disease: a mixed treatment comparison. CNS Neurosci Ther. 2017;23(10):827-42.

5. Brundin P, Barker RA, Conn PJ, Dawson TM, Kieburtz K. Linked clinical trials the development of new clinical learning studies in Parkinson's disease using screening of multiple prospective new treatments. J Park Dis. 2015; 3(3):231-9.

6. Spielman LJ, Little JP, Klegeris A. Physical activity and exercise attenuate neuroinflammation in neurological diseases. Brain Res Bull. 2016;125:19-29.

7. World Health Organization. Health topics 2018 [Internet]. [cited $2018 \mathrm{Mar}$ 29]. Available from: http://www.who.int/topics/physical_activity/en/.

8. Alkadhi KA. Exercise as a positive modulator of brain function. Mol Neurobiol. 2017;55(4):3112-30.

9. Mantri S, Fullard ME, Duda JE, Morley JF. Physical activity in early Parkinson disease. J Parkinsons Dis. 2018;8(1):107-11.

10. Ahlskog JE. Aerobic exercise: evidence for a direct brain effect to slow Parkinson disease progression. Mayo Clin Proc. 2018;93(3):360-72.

11. Zhou W, Barkow JC, Freed CR. Running wheel exercise reduces alphasynuclein aggregation and improves motor and cognitive function in a transgenic mouse model of Parkinson's disease. PLoS One. 2017;12(12): e0190160.

12. Klemann CJHM, Xicoy H, Poelmans G, Bloem BR, Martens GJM, Visser JE. Physical exercise modulates L-DOPA-regulated molecular pathways in the MPTP mouse model of Parkinson's disease. Mol Neurobiol. 2018; 55(7):5639-57.

13. Jang Y, Kwon I, Song W, Cosio-Lima LM, Taylor S, Lee Y. Modulation of mitochondrial phenotypes by endurance exercise contributes to neuroprotection against a MPTP-induced animal model of PD. Life Sci. 2018 Aug;209:455-65.

14. Jang YC, Hwang DJ, Koo JH, Um HS, Lee NH, Yeom DC, et al. Association of exercise-induced autophagy upregulation and apoptosis suppression with neuroprotection against pharmacologically induced Parkinson's disease. J Exerc Nutr Biochem. 2018;22(1):1-8.

15. Blesa J, Trigo-Damas I, Quiroga-Varela A, Jackson-Lewis VR. Oxidative stress and Parkinson's disease. Front Neuroanat. 2015;9:1-9.

16. Smeyne RJ, Jackson-Lewis V. The MPTP model of Parkinson's disease. Mol Brain Res. 2005;134(1):57-66.

17. Carradori S, Ortuso F, Petzer A, Bagetta D, De Monte C, Secci D, et al. Design, synthesis and biochemical evaluation of novel multi-target inhibitors as potential anti-Parkinson agents. Eur J Med Chem. 2018;143: 1543-52.

18. Shungu DC. Clinical Trials.gov [Internet]. N-Acetylcysteine for Neuroprotection in Parkinson's Disease (NAC for PD). 2017 [cited 2018 Mar 30]. Available from: https:/clinicaltrials.gov/ct2/show/study/ NCT01470027?term=N-acetyl-cysteine\&cond=Parkinson+Disease\&rank=2

19. Pan J, Xiao Q, Sheng CY, Hong Z, Yang HQ, Wang G, et al. Blockade of the translocation and activation of c-Jun N-terminal kinase 3 (JNK3) attenuates dopaminergic neuronal damage in mouse model of Parkinson's disease. Neurochem Int. 2009;54(7):418-25.

20. Barcia C, Ros CM, Annese V, Gómez A, Ros-Bernal F, Aguado-Yera D, et al. IFN- $\gamma$ signaling, with the synergistic contribution of TNF-a, mediates cell specific microglial and astroglial activation in experimental models of Parkinson's disease. Cell Death Dis. 2011;2:e142.

21. Barcia C, Ros CM, Annese V, Carrillo-de Sauvage MA, Ros-Bernal F, Gómez A, et al. ROCK/Cdc42-mediated microglial motility and gliapse formation lead to phagocytosis of degenerating dopaminergic neurons in vivo. Sci Rep. 2012;2:809.

22. Jackson-Lewis $\mathrm{V}$, Przedborski S. Protocol for the MPTP mouse model of Parkinson's disease. Nat Protoc. 2007;2(1):141-51.

23. Franklin K, Paxinos G. The Mouse Brain in Stereotaxic Coordinates. 2nd ed; 2004.

24. Blesa J, Pifl C, Sánchez-González MA, Juri C, García-Cabezas MA, Adánez R, et al. The nigrostriatal system in the presymptomatic and symptomatic stages in the MPTP monkey model: a PET, histological and biochemical study. Neurobiol Dis. 2012;48(1):79-91.

25. Annese V, Herrero MT, Di Pentima M, Gomez A, Lombardi L, Ros CM, et al, Metalloproteinase-9 contributes to inflammatory glia activation and nigrostriatal pathway degeneration in both mouse and monkey models of 1 methyl-4-phenyl-1,2,3,6-tetrahydropyridine (MPTP)-induced parkinsonism. Brain Struct Funct. 2015;220(2):703-27.

26. Carrillo-de Sauvage MÁ, Maatouk L, Arnoux I, Pasco M, Sanz Diez A, Delahaye $\mathrm{M}$, et al. Potent and multiple regulatory actions of microglial glucocorticoid receptors during CNS inflammation. Cell Death Differ. 2013; 20(11):1546-57.

27. Samson M, Porter N, Orekoya O, Hebert JR, Adams SA, Bennett CL, et al. Glial Cell Morphological and Density Changes Through the Lifespan of Rhesus Macaques. Brain Behav Immun. 2017;155(1):3-12.

28. Tatsumi K, Okuda H, Morita-Takemura S, Tanaka T, Isonishi A, Shinjo T, et al. Voluntary exercise induces astrocytic structural plasticity in the globus pallidus. Front Cell Neurosci. 2016;10:1-12.

29. Huang D, Xu J, Wang J, Tong J, Bai X, Li H, et al. Dynamic changes in the nigrostriatal pathway in the MPTP mouse model of Parkinson's disease. Parkinsons Dis. 2017;2017:9349487.

30. Barcia C, Bautista V, Sánchez-Bahillo A, Fernández-Villalba E, Navarro-Ruis JM, Fernández Barreiro A, et al. Circadian determinations of cortisol, prolactin and melatonin in chronic methyl-phenyl-tetrahydropyridine-treated monkeys. Neuroendocrinology. 2003;78(2):118-28.

31. Barcia C, De Pablos V, Bautista-Hernández V, Sanchez-Bahillo A, FernándezBarreiro A, Poza M, et al. Measurement of motor disability in MPTP-treated macaques using a telemetry system for estimating circadian motor activity. J Neurosci Methods. 2004;134(1):59-64. 
32. Wang Q, Liu Y, Zhou J. Neuroinflammation in Parkinson's disease and its potential as therapeutic target. Transl Neurodegener. 2015;4(1):19.

33. McGeer PL, McGeer EG. Glial reactions in Parkinson's disease. Mov Disord. 2008;23(4):474-83.

34. Miller RL, James-Kracke M, Sun GY, Sun AY. Oxidative and inflammatory pathways in parkinson's disease. Neurochem Res. 2009;34(1):55-65.

35. Jang Y, Koo J-H, Kwon I, Kang E-B, Um H-S, Soya H, et al. Neuroprotective effects of endurance exercise against neuroinflammation in MPTP-induced Parkinson's disease mice. Brain Res. 2016;1655:186-93.

36. Halliday GM, Stevens CH. Glia: initiators and progressors of pathology in Parkinson's disease. Mov Disord. 2011;26(1):6-17.

37. Liddelow SA, Guttenplan KA, Clarke LE, Bennett FC, Bohlen CJ, Schirmer L, et al. Neurotoxic reactive astrocytes are induced by activated microglia. Nature. 2017;541(7638):481-7.

38. Batlle M, Ferri L, Andrade C, Ortega FJ, Vidal-Taboada JM, Pugliese M, et al. Astroglia-microglia cross talk during neurodegeneration in the rat hippocampus. Biomed Res Int. 2015;2015:102419.

39. Kettenmann H, Hanisch U-K, Noda M, Verkhratsky A. Physiology of microglia. Physiol Rev. 2011;91(2):461-553.

40. Sofroniew MV, Vinters HV. Astrocytes: biology and pathology. Acta Neuropathol. 2010;119(1):7-35.

41. Edwards MM, Robinson SR. TNF alpha affects the expression of GFAP and S100B: implications for Alzheimer's disease. J Neural Transm. 2006; 113(11):1709-15.

42. Steiner J, Bernstein HG, Bielau H, Berndt A, Brisch R, Mawrin C, et al. Evidence for a wide extra-astrocytic distribution of $\mathrm{S100B}$ in human brain. BMC Neurosci. 2007:8:1-10.

43. Himeda T, Watanabe Y, Tounai H, Hayakawa N, Kato H, Araki T. Time dependent alterations of co-localization of S100B and GFAP in the MPTPtreated mice. J Neural Transm. 2006;113(12):1887-94.

Ready to submit your research? Choose BMC and benefit from:

- fast, convenient online submission

- thorough peer review by experienced researchers in your field

- rapid publication on acceptance

- support for research data, including large and complex data types

- gold Open Access which fosters wider collaboration and increased citations

- maximum visibility for your research: over $100 \mathrm{M}$ website views per year

At $\mathrm{BMC}$, research is always in progress.

Learn more biomedcentral.com/submissions 